\title{
Reversed dimensional analysis in psychophysics
}

\author{
S. ALEX MARINOV \\ Applied Linguistics Centre and SAM Research, Winnipeg, Manitoba, Canada
}

\begin{abstract}
It is argued that dimensional analysis (from physics) can be applied in psychophysics in the same manner as in physics, provided that the dimensions of sensations are interpreted in the sense of reversed dimensional analysis, which allows for extracting dimensions from data. A dimension obtained by this method, referred to as phenomenological dimension, is similar to a fractal dimension. The examples discussed show that, if the dimension of sensation in Stevens' psychophysical law for time duration ( $\left.\Psi=C \Phi^{n}, n \oplus 1.15\right)$ is interpreted as a phenomenological dimension, such a dimension can be traced even in short-term memory data. It is suggested that dimensions obtained via reversed dimensional analysis may serve as a link between physical and psychological variables and as a basis for developing the notion of dimension in psychology.
\end{abstract}

\section{Dimensional Analysis and \\ Reversed Dimensional Analysis}

Dimensional analysis (DA) in physics is based on the presumption that physical laws do not depend on the choice of measurement scales and units (Barenblatt, 1987; Bridgman, 1922; Krantz, Luce, Suppes, \& Tversky, 1971). A physical equation shall be dimensionally homogeneous - that is, the left and the right sides of such an equation shall have the same physical dimension. Physical dimensions are thought of as denotations (symbols or names) of qualitatively distinguishable properties of physical objects (Ellis, 1966; Krantz et al., 1971; Munson, Young, \& Okiishi, 1990; Pankhurst, 1964) and are defined prior to measurement. It is commonly agreed that physical dimensions can be divided into two groups: (1) primary, also referred to as basic or fundamental dimensions, and (2) secondary, or derived, dimensions. Depending on the choice of measurement units, different sets of dimensions can be selected as primary dimensions. All other dimensions can be considered to be secondary dimensions. For example, in Systeme International, the dimensions of length $(L)$, mass $(M)$, and time $(T)$ are considered to be a complete set of primary dimensions in mechanics. The dimensions of such quantities as velocity $\left(L / T\right.$ or $\left.L^{1} T^{-1}\right)$ and force $\left(L M / T^{2}\right.$ or $\left.L^{1} M^{1} T^{-2}\right)$ are considered to be secondary dimensions. Any secondary dimension can be expressed as a product of power functions of the primary dimensions. For in-

I thank Daniel Algom, John Baird, Neil Macmillan, Kenneth Norwich, and Lawrence Ward for their valuable comments, Tara Callaghan for her comments and assistance in the preparation of an early version of this article, David Laurence and Konstantin Gubarev for their assistance in the preparation of a later version, and especially Julia Boon for her continuous support of this study. Correspondence concerning this article should be addressed to S. A. Marinov, Applied Linguistics Centre, 575 Wall Street, Winnipeg, MB, R3G 2T5 Canada (e-mail: marinov@ escape.ca).

Note-This article was accepted by the previous editorial team, headed by Neil Macmillan. stance, all secondary dimensions in mechanics can be expressed in the form $L^{\lambda} M^{\mu} T^{\tau}$, where the exponents are small integers: -4 " $\lambda^{\prime \prime}+4,-1$ " $\mu^{\prime \prime}+1$, and -3 " $\tau^{\prime \prime}$ +2 (Krantz et al., 1971). Square brackets ([ ] ) are commonly used to denote the dimension of a quantity.

DA has proven its efficiency as a powerful tool in almost all branches of physics, as well as in such disciplines as engineering and chemistry (Barenblatt, 1987; Bridgman, 1922; Sedov, 1981). Applications of DA range from simple error checks of physical equations to obtaining explicit functional relations among variables relevant to a phenomenon being investigated. DA is commonly used in reducing the number of the initial set of dimensional variables to a smaller number of dimensionless variables, using the П-theorem (Buckingham, 1914).

\section{П-theorem}

If the relation among $m$ dimensional variables (including dimensional constants) is

$$
f\left(X_{1}, X_{2}, \ldots X_{m}\right)=0,
$$

where $X_{1}, X_{2}, \ldots X_{m}$ is a complete set of dimensional variables and constants relevant to the phenomenon being investigated, such a relation can be reduced to a relation among $m-k$ dimensionless variables,

$$
F\left(\Pi_{1}, \Pi_{2}, \ldots \Pi_{m-k}\right)=0,
$$

where $k$ is the number of dimensional variables and constants with independent dimensions and all П-terms are dimensionless products of powers of the dimensional variables/constants,

$$
\Pi_{i}=X_{1}^{p_{i 1}} X_{2}^{p_{i 2}}, \ldots X_{m}^{p_{\text {in }}} . \quad(i=1, \ldots k) .
$$

It is said that a variable has independent dimension if its dimension cannot be obtained from the dimensions of the other variables. For example, if $f\left(l_{1}, l_{2}, t, v\right)=0 \mathrm{de}$ scribes a problem involving four variables, where the dimensions of the variables are $\left[l_{1}\right]=L,\left[l_{2}\right]=L,[t]=T$, 
and $[v]=L / T, l_{1}$ and $t$ can be considered as variables with independent dimensions, whereas $l_{2}$ and $v$ can be considered as variables with dependent dimensions. The choice of independent/dependent dimensions is not an absolute one but, rather, a matter of convenience. Obviously, in the case of $f\left(l_{1}, l_{2}, t, v\right)=0$, another couple of variables - say, $l_{2}$ and $t$ - can be chosen as variables with independent dimensions, whereas the other two variables can be considered as variables with dependent dimensions. DA, including the $\Pi$-theorem, is based on the assumption that the magnitudes of the quantities in physics are ratio scaled (Bridgman, 1922). DA imposes no restriction on the form of the function $F\left(\Pi_{1}, \Pi_{2}, \ldots\right.$ $\left.\Pi_{m-k}\right)=0$ but only on the form of the arguments $\Pi_{1}, \Pi_{2}$, $\ldots \Pi_{m-k}$. The explicit form of $F$ cannot be found by means of DA alone, except for the special case of $m-k=1$, which results in $\Pi_{1}=C$, where $C$ is a dimensionless constant.

Limited attempts at applying DA have been made in other fields, such as economics (De Jong, 1967) and physical geography (Haynes, 1982). DA has enjoyed certain attention in psychology and, especially, in psychophysics, primarily due to the work of Luce (Krantz et al., 1971; Luce, 1959, 1964, 1971, 1978, 1990). However, these studies were rather theoretical, which may be the reason that Laming (1997) recently wrote: "While the argument from DA might be justifiable as an exercise in axiomatization, it contributes nothing to psychophysics" (p. 12). In fact, in order to apply DA to certain problems, one must know the dimensions of all the variables and constants involved. Although this is not an issue in physical applications, it appears to be a fundamental obstacle beyond physics, since some of the variables do not have a dimension in the sense in which it is thought of in physics. The problem is, therefore, not the limited power of DA per se but the lack of clear understanding of what a dimension of a nonphysical variable is.

A possible solution to this problem is the method of reversed dimensional analysis (RDA; Marinov, 1994; Marinov \& Panov, 2000). In this approach, if a dimension cannot be assigned to a variable in the way in which this takes place in physics but such a variable is yet measurable, the dimension of this variable can be calculated from data, provided that measurement data are available. Such a concept of dimension (quantity calculated a posteriori from data) is fundamentally different from the concept of dimension in physics (qualitative denotation attributed a priori to a physical property). A dimension obtained by the method of RDA, referred to as a phenomenological dimension, is similar in nature to a fractal dimension (Mandelbrot, 1977, 1983). The latter notion of dimension is now widely recognized in mathematics and science. Unlike physical dimensions (specifically, the dimensions of length $L^{1}$, area $L^{2}$, and volume $L^{3}$ ), a fractal dimension is a dimension of the kind $L^{\lambda^{*}}$, where $\lambda^{*}$ is a small number that (1) need not be an integer, (2) is normally calculated from data, and (3) may reflect a qualitatively specific state of the object/phenomenon being investigated.Fractal dimensions are used in the description of geometrical properties of highly irregular objects. It can be shown that a fractal dimension is, in fact, a special case of a phenomenological dimension, in which only geometrical properties are considered (Marinov, 2001).

\section{APPLICATION OF RDA IN PSYCHOPHYSICS}

Broadly defined, psychophysics is a study of the relationship between the physical stimulus and the subject's response to that stimulus (cf. Goldstein, 1989). The former is usually a well-defined physical quantity, such as time, length, mass, or light intensity. The subject's response, frequently referred to as a sensory response, is in fact a final result of a cascade of complex processes, including stimulus-receptor interaction, peripheral processing, central processing, sensory response per se, perceptual response, and decision/judgment (Marks, 1992). Since psychophysics was established as a discipline (Fechner, 1860/1966), sensations have been considered by most psychophysicistsas quantitativeentities (but see Michell, 1997). To our knowledge, however, the question about the dimensions of such quantities has never been raised. It appears, rather, that different measurement procedures result in different "dimensions" of the same sensory quantity.

Consider Stevens' psychophysical law. For so-called prothetic continua (Stevens, 1959), the following law holds: $\Psi=C \Phi^{n}$, where $\Psi$ is the magnitude of sensation, $\Phi$ is the stimulus magnitude, and both $C$ and $n$ are parameters, also referred to as constants, that differ for different sensations (Stevens, 1975). Depending on the measurement procedure, $\Psi$ can be reported either in terms of a dimensionless number or in terms of the physical dimension of the stimulus. In both cases, the problem of the dimension of the particular sensation is camouflaged by the dimensional constant $C$. Unlike the exponent $n$, the constant $C$ is viewed as an entity of secondary importance, a coefficient devoid of psychological meaning (Baird, 1997).

If $\Psi$ is reported as a dimensionless number, the dimension of $C$ is $[C]=[\Phi]^{-n}$. (Recall that brackets are used to denote the dimension of the corresponding quantity.) On the other hand, if $\Psi$ is reported as a quantity with the dimension of the stimulus, the dimension of the constant $C$ is $[C]=[\Phi]^{1-n}$. Either interpretation serves to dimensionally homogenize the law, but the nature of this constant is unclear, and it does not contribute to the understanding of the mechanisms of sensation. According to Bridgman (1922), such dimensional constants are to be strongly avoided.

To make the discussion concrete, consider estimation of time intervals. In this case, $\Psi$ is the response of the subject either in terms of dimensionless numbers or in terms of time duration (seconds, minutes, etc.). $\Phi$ is the stimulus duration measured in certain physical units of time. In other words, the dimension of $\Psi$ is either $[\Psi]=1$ 
(dimensionless) or $[\Psi]=T$ (dimension of time), whereas the dimension of $\Phi$ in both cases is $[\Phi]=T$. We call these alternatives Case 1 and Case 2. In order to maintain the dimensional homogeneity of the law, the dimension of the constant $C$ has to be $[C]=[\Phi]^{-n}=T^{-n}$ or $[C]=$ $[\Phi]^{1-n}=T^{1-n}$, respectively. The commonly accepted value of the exponent in Stevens' law for time duration is $n \approx 1.15$ (Stevens, 1959).

Table 1 summarizes Case 1 and Case 2, which represent what can be reported in a psychophysical experiment, and Case 3, which is essentially different. It represents a situation in which no dimensional constant with unclear dimension is introduced, and the problem of unclear dimensionality is transferred to the variable $\Psi$. A dimension of time $(T)$ raised to the power of a noninteger number $(n \approx 1.15)$ - that is, $T^{1.15}$-is attributed to the quantity regarded as "sensation" in estimating time intervals.

In addition to obtaining the functional relation between $\Phi$ and $\Psi$, Case 3 also results in assigning a dimension to the sensation $\Psi$. As was mentioned above, dimensions are viewed as characteristics that reflect qualitatively distinguishable properties of an object. It is, therefore, assumed that such properties are inherent to the object and are generally preserved when the object interacts with other objects. As was also mentioned, even in the case of fractal dimensions, the particular value of such a dimension may reflect a qualitatively distinguishable state of the object/phenomenon being investigated. Therefore, although the functional relation may change from case to case, the dimension of the quantity will not. Thus, the dimension of a quantity is a more fundamental entity than the form of the functional relation the quantity is involved in. In fact, except for some exotic cases (cf. Collins, 1984), the entire science of physics is an example of dealing with quantities that preserve their dimensions while entering into different functional relations.

A problem related to Case 3 arises from the obvious fact that the subject cannot be asked to estimate time intervals in terms of a quantity whose dimension is not known prior to measurement. If, however, for data obtained in an experiment corresponding to Case 1, the sensation $\Psi$ is considered as a quantity with unknown dimension and the constant $C$ is considered as dimensionless, such a combination of dimensions will correspond to Case 3, where the dimension of $\Psi$ can be obtained by

Table 1

Three Possible Combinations of Dimensions That Preserve the Dimensional Homogeneity of Stevens' Psychophysical Law $\left(\Psi=C \Phi^{n}\right)$ for Time Duration

\begin{tabular}{cccc}
\hline Case & $\Psi$ & $C$ & $\Phi$ \\
\hline 1 & 1 & $T^{-n}$ & $T$ \\
2 & $T$ & $T^{1-n}$ & $T$ \\
3 & $T^{n}$ & 1 & $T$ \\
\hline
\end{tabular}

Note-The symbol $T$ stands for the dimension of time, whereas 1 stands for the dimension of a dimensionless quantity. the method of RDA. In this case, $[\Psi]=[\Phi]^{n}=T^{n}$, where the exponent $n$ is obtained via regression of experimental data in exactly the same way as in Stevens' psychophysical law for time duration.

Will the phenomenological dimensions of variables of the same nature (e.g., those related to time intervals) be the same in different psychological tasks? In the following sections, we will examine this question. It will be shown that the phenomenological dimension of a timerelated quantity reported in a short-term memory experiment is practically the same as the phenomenological dimension of the quantity regarded as "sensation" in Stevens' psychophysical law for time duration.

\section{APPLICATION OF RDA TO DATA OBTAINED IN SHORT-TERM MEMORY EXPERIMENTS}

\section{Analysis of Hellyer's (1962) Data}

In an experiment of the same type as that in Peterson and Peterson (1959), Hellyer (1962) studied the proportion of items (consonant trigrams) correctly recalled (the $X_{1}$ variable) as a function of two variables: recall delay interval (the $X_{2}$ variable) and number of presentations (the $X_{3}$ variable). The relation between these three variables can be expressed as

$$
X_{1}=f\left(X_{2}, X_{3}\right),
$$

where, according to Hellyer, the dimensions of the variables are $\left[X_{1}\right]=1$ (dimensionless), $\left[X_{2}\right]=T$ (dimension of time), and $\left[X_{3}\right]=1$ (dimensionless). Apparently, this relation is dimensionally inhomogeneous unless at least one dimensional constant-say $C_{H}$-with a dimension of the kind $\left[C_{H}\right]=T^{\tau}$ is introduced, where the exponent $\tau$ will depend on the explicit form of Equation 1. An approximation to the explicit form of this relation can be found via regression analysis, provided measurement data are available. Thus, an analogue of Table 1, omitting Case 2, is Table 2.

Case 1 in Table 2 corresponds to the original interpretation suggested by Hellyer (1962) - that is, $X_{1}=f\left(X_{2}\right.$, $X_{3}$ ) - and implicitly assumes the introduction of the dimensional constant $C_{H}$. Case 3 assumes no dimensional constant, but the dimension of the third variable $\left(X_{3}\right)$ is interpreted as a phenomenological dimension. According to the $\Pi$-theorem of DA, if the three variables $X_{1}, X_{2}$, and $X_{3}$ are the only ones relevant to the phenomenon being investigated, the relation in Equation 1 can be reduced to a relation between two dimensionless variables $\left(\Pi_{1}\right.$ and $\left.\Pi_{2}\right)$ :

$$
\Pi_{1}=F\left(\Pi_{2}\right)
$$

Taking into account the dimension of the variables (see Table 2, Case 3), the $\Pi$-terms are

$$
\Pi_{1}=X_{1}
$$

and

$$
\Pi_{2}=X_{2}^{p_{22}} X_{3}^{p_{23}}
$$


Table 2

Two Possible Combinations of Dimensions That Preserve the Dimensional Homogeneity of the Functional Relation Studied by Hellyer (1962)

\begin{tabular}{ccccc}
\hline & $\begin{array}{c}\text { Proportion of Items } \\
\text { Correctly Recalled }\end{array}$ & $\begin{array}{c}\text { Dimensional } \\
\text { Constant } \\
\left(X_{H}\right)\end{array}$ & $\begin{array}{c}\text { Recall Delay } \\
\text { Interval } \\
\left(X_{2}\right)\end{array}$ & $\begin{array}{c}\text { Number of } \\
\text { Presentations } \\
\left(X_{3}\right)\end{array}$ \\
\hline 1 & 1 & $T^{\tau}$ & $T$ & 1 \\
3 & 1 & 1 & $T$ & $T^{\tau}$ \\
\hline
\end{tabular}

Note-Case 1 assumes the introduction of a dimensional constant $\left(C_{H}\right)$, whereas Case 3 interprets the dimension of the third variable $\left(X_{3}\right)$ as a phenomenological dimension, which, in this particular case, depends on the dimension of the other dimensional variable $\left(X_{2}\right)$.

As is shown in the Appendix, $\Pi_{2}$ can be redefined (e.g., by raising both sides to the power of $1 / p_{23}$ ) so that only one exponent will appear on the right side:

$$
\Pi_{1}=X_{1}
$$

and

$$
\Pi_{2}=X_{2}^{p} X_{3} .
$$

Taking into account Equation 4, the expression in Equation 3 can be rewritten in the form

$$
X_{1}=F\left(X_{2}^{p} X_{3}\right)
$$

where the relation between the dimensions of the two variables $X_{2}$ and $X_{3}$ is given by the expression

$$
\left[X_{3}\right]=\left[X_{2}\right]^{-p}=T^{-p} \text {. }
$$

In other words, if the number of presentations variable $\left(X_{3}\right)$ in Hellyer's (1962) data is considered as a variable with unknown dimension, this dimension will depend on the dimension of the only other dimensional variable, recall delay interval $\left(X_{2}\right)$, according to the expression in Equation 6 . The exponent $p$ is an unknown number, which, however, can be found by the method of RDA if measurement data for all three variables $\left(X_{1}, X_{2}\right.$, and $\left.X_{3}\right)$ are available. Such data were reported by Hellyer, and are plotted in Figure 1.

As is described in detail in the Appendix, the numerical value of the exponent $p$ can be found by fitting Equation 2, respectively Equation 5, with an appropriate function. In such a case, the unknown exponent $p$ is con- sidered as a regression parameter, along with the conventional regression parameters, which are specific for each fitting function. The result of testing five different fitting functions is shown in Table 3. The coefficient of variation for regression residuals $(C V$; cf. Harnett \& Murphy, 1993) was used as a goodness-of-fit criterion. In addition to the point values of $p$ corresponding to $C V_{\min }(p)$ for each of the fitting functions, we also calculated $C V(p)$ within the interval $-10 \leq p \leq+10$, using grid steps $\Delta p=1,0.1,0.01$, and 0.001 . The curves $C V(p)$ corresponding to each of the five fitting functions are plotted in Figure 2. This approach allows one to find all local minima within the given interval, as well as to numerically estimate the sharpness of any minimum by the means of a ratio of the kind $\left(C V-C V_{\min }\right) / C V_{\min }$. On the other hand, the same kind of ratio can be used to estimate the accuracy of determining $p$ in terms of an interval value $p^{-} \leq p_{\min } \leq p^{+}$, where $p^{-}$and $p^{+}$correspond to $\left(C V-C V_{\min }\right) / C V_{\min }=\delta^{*}$ on the left and the right sides of the minimum, respectively, and $\delta^{*}$ is a small number chosen a priori.

According to Table 3, the smallest value of the coefficient of variation $(\mathrm{CV})$ corresponds to the power exponential function, and therefore, this function provided the best fit among the five regression functions tested. The value of $p$ that corresponds to this function is $p_{\min }=$ -1.13 . (Recall that the exponent in a phenomenological dimension, as in the case of a fractal dimension, need not be an integer.) On the basis of the ratio introduced in the

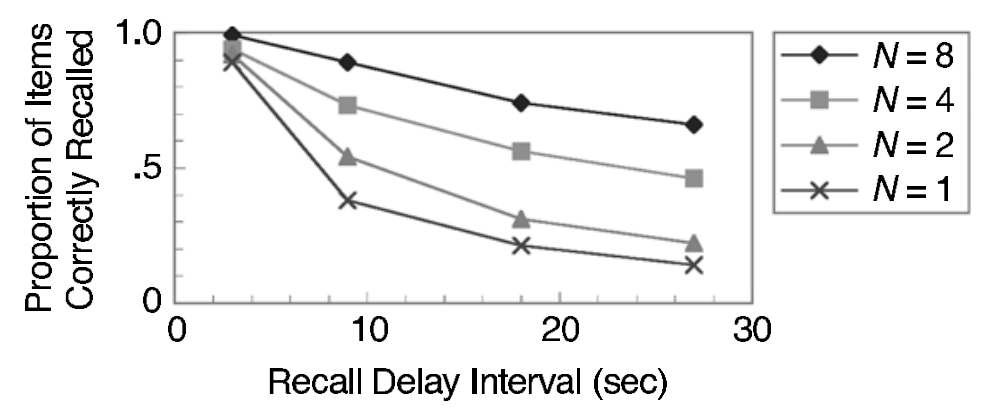

Figure 1. Proportion of items (consonant trigrams) correctly recalled as a function of recall delay interval and number of presentations $(N)$. Data are from Hellyer (1962). 
Table 3

Values of the Exponent ( $p$ ) Corresponding to the Minimum Values of the Coefficient of Variation, $C V_{\min }=C V\left(p_{\min }\right)$, for Five Different Regression Functions

\begin{tabular}{llcc}
\hline Regression Function & \multicolumn{1}{c}{ Expression** } & $C V_{\min }$ & $p_{\min }$ \\
\hline Linear & $A+B \cdot \Pi_{2}$ & 0.33473 & -0.538 \\
Power & $A \cdot \Pi_{2}^{B}$ & 0.29619 & -3.399 \\
Exponential & $A \cdot e^{B \cdot \Pi_{2}}$ & 0.41220 & -0.177 \\
Power exponential* & $A \cdot \Pi_{2}^{B} \cdot e^{C \cdot \Pi_{2}}$ & 0.14778 & -1.130 \\
Quadratic & $A+B \cdot \Pi_{2}+C \cdot \Pi_{2}^{2}$ & 0.21449 & -0.904 \\
\hline
\end{tabular}

Note-Interval of seeking minima: $-10 \leq p \leq+10$. Grid step: $\Delta p=$ 0.001. Analysis of Hellyer's (1962) data. *Also referred to as a Hoerl's special function (Daniel \& Wood, 1971). **A, $B$, and $C$ are constants that differ for each function.

previous paragraph, if for instance $\delta^{*}=1 \%$, the interval estimate of $p_{\min }$ will be $-1.21 \leq p_{\min } \leq-1.06$. In order to obtain the phenomenological dimension of variable $X_{3}, p_{\text {min }}=-1.13$ shall be substituted into Equation 6:

$$
\left[X_{3}\right]=\left[X_{2}\right]^{-p}=T^{-p}=T^{-(-1.13)}=T^{1.13} .
$$

Thus, according to Equation 7, the phenomenological dimension of the number of presentations variable in Hellyer's (1962) data is practically the same as the phenomenological dimension of the quantity regarded as "sensation" in Stevens' psychophysical law for time duration $\left(T^{1.15}\right)$. In other words, in two essentially different psychological experiments (magnitude estimation and memory recall), it was found that variables related to processing of time exhibit the same phenomenological dimensions.

It can reasonably be argued that the variable $X_{3}$ (number of presentations) is an external independent variable and, therefore, has nothing to do with the way time intervals are processed by the subject. With respect to the variable per se, this is correct. Recall, however, that the computation of the phenomenological dimension of this variable involves all three variables, including $X_{1}$ (proportion of items correctly recalled), which is, in fact, the measure of the response of the subject. As is shown in Figure 1, this response is affected by both external variables $X_{2}$ and $X_{3}$. In other words, the time intervals involved in Hellyer's (1962) experiment essentially affect the way the subject recalls and, presumably, memorizes items. This, however, does not necessarily imply that these time intervals are processed by the subject in the same manner as those in a time interval estimation experiment. Are the time intervals in Hellyer's experiment, then, just passive frames of the process of memorizing and recalling items, or are they actively processed, since this presumably takes place in a psychophysical experiment, where the only task is estimating the intervals? Or is the answer in between these two marginal statements? To our knowledge, such a question has not yet been answered. What, however, can certainly be concluded within the framework of RDA is that processing of time appears to exhibit self-similarity with respect to the time scales involved in time interval estimation and memory recall (based on Hellyer's data).

The concept of self-similarity is best illustrated in terms of the spatial properties of objects. An object is selfsimilar if the object, when every part is magnified by a suitable scale factor, is identical to the original object. Scale invariance is a related and stronger property, which is thought of as self-similarity on all scales (Creswick, Farach, \& Poole, 1992). Both concepts can be generalized with respect to other properties of objects/phenomena.

Is self-similarity with respect to psychological time scales always the case, so that it could be claimed that the processing of time is scale invariant? At this time, the relatively limited amount of psychological data processed by RDA does not really allow for a definitive answer. Yet apart from the almost identical phenomenological dimensions discussed above, there is some evidence that RDA produces rather similar results for data obtained in similar conditions. On the other hand, it allows us to distinguish data obtained in seemingly similar conditions that, in fact, differ essentially. The following analysis of

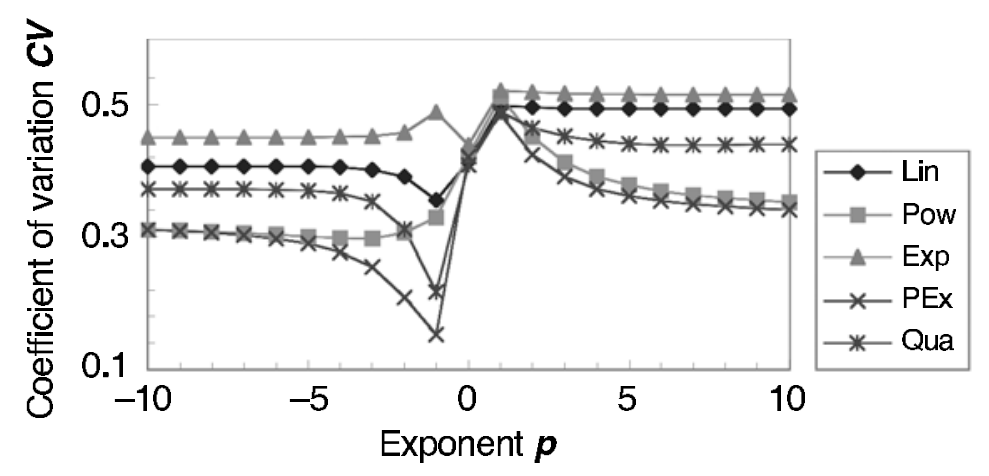

Figure 2. The coefficient of variation for regression residuals $C V$ as a function of the exponent $p$. Regression functions: linear (Lin), power (Pow), exponential (Exp), power exponential (PEx), and quadratic (Qua). Interval of seeking minima: $-10 \leq p \leq+10$. Grid step: $\Delta p=1$. The analysis is of Hellyer's (1962) data. 
data reported by Peterson and Peterson (1959) illustrates this statement. It is to be noted that Hellyer's (1962) experiment, discussed above, was a slightly modified replication of Peterson and Peterson's original experiment. Both studies have enjoyed extensive citation and reanalysis since they were published about 40 years ago (see Laming, 1992, and references therein).

\section{Analysis of Peterson and Peterson's (1959) Data}

Peterson and Peterson (1959) reported three groups of data involving variables similar in nature to those reported by Hellyer (1962): the proportions of items (consonant trigrams) correctly recalled $\left(X_{1}\right)$ as a function of two variables-recall delay interval $\left(X_{2}\right)$ and number of repetitions $\left(X_{3}\right)$. Hellyer's and Peterson and Peterson's experiments differ in two major aspects: (1) visual stimulus presentation versus verbal and (2) multiple presentation versus single presentation. In addition, Peterson and Peterson divided the subject pool into two groups. Half of the subjects (regarded as the vocal group) were instructed to repeat the stimulus aloud following 1 -sec ticks of a metronome in the interval between stimulus presentation and the moment of starting backward counting as a distracting activity during recall delay. The other half (regarded as the silent group) were not given instructions concerning their activity within this interval. In contrast, all of Hellyer's subjects were given instructions to repeat aloud a repeatedly displayed stimulus (display rate, $1 / \mathrm{sec}$ ). In addition to the vocal and silent data, Peterson and Peterson reported a third data set corresponding to the same three variables $\left(X_{1}, X_{2}\right.$, and $X_{3}$ ). However, in this case, $X_{1}$ stands for the probability of a letter's being correctly recalled in the vocal group when the preceding letter was correct (Peterson \& Peterson, 1959, p. 197). Thus, the relationship among the three variables $\left(X_{1}, X_{2}\right.$, and $\left.X_{3}\right)$ can be expressed as a set of three equations corresponding to each of the three data sets reported by Peterson and Peterson:

$$
\begin{aligned}
& X_{1}^{\text {vocal }}=f^{\text {vocal }}\left(X_{2}, X_{3}\right), \\
& X_{1}^{\text {silent }}=f^{\text {silent }}\left(X_{2}, X_{3}\right),
\end{aligned}
$$

and

$$
X_{1}^{\text {letter }}=f^{\text {letter }}\left(X_{2}, X_{3}\right) .
$$

The expression in Equation 8 is a complete analogue of that in Equation 1, except for the three different conditions. The data corresponding to each of these conditions were analyzed in the same manner, including the use of the same five regression functions, as was done in the case of Hellyer's data. Figure 3 shows the results of applying RDA to the three data sets reported by Peterson and Peterson, along with the results of analyzing Hellyer's data.

The first observation is that the curves corresponding to four different data sets are all U-shaped, so that $p_{\min }$ exists in all cases. This is not a corollary of the method of RDA per se. To the contrary, both the model experi-
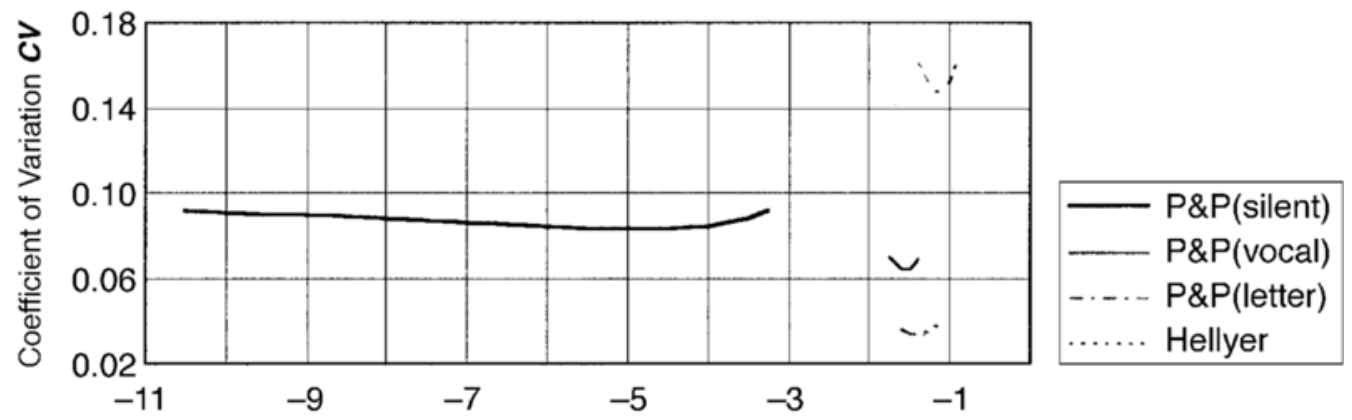

Exponent $\boldsymbol{p}$
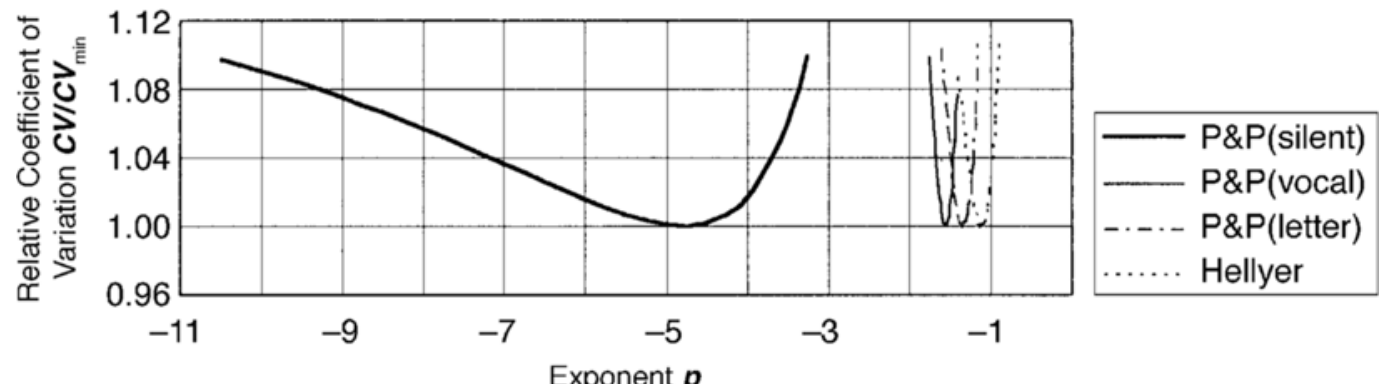

Figure 3. The coefficient of variation for regression residuals $(C V$, top $)$ and the relative coefficient of variation for regression residuals $\left(C V / C V_{\min }\right.$, bottom) as functions of the exponent $p$. Fitting function: power exponential (PEx). Interval of seeking minima: $-10 \leq p \leq+10$. Grid step: $\Delta p=0.001$. The analysis is of Peterson and Peterson's (1959) and Hellyer's (1962) data. 
ments and the analysis of real data show that there exist cases in which $C V(p)$ does not have a minimum within intervals of seeking minima as large as $-20 \leq p \leq+20$. On the basis of the data plotted in Figure 3, the following phenomenological dimensions, corresponding to each of the three conditions, were calculated:

$$
\begin{aligned}
& {\left[X_{3}\right]_{(\text {vocal })}=\left[X_{2}\right]^{-p_{(\text {vocal })}}=\left[X_{2}\right]^{1.546}=T^{1.546} \approx T^{1.55},} \\
& {\left[X_{3}\right]_{(\text {silent })}=\left[X_{2}\right]^{-p_{(\text {silent })}=\left[X_{2}\right]^{4.798}=T^{4.798} \approx T^{4.80},}}
\end{aligned}
$$

and

$$
\left[X_{3}\right]_{(\text {letter })}=\left[X_{2}\right]^{-p_{\text {(leter) }}}=\left[X_{2}\right]^{1.355}=T^{1.355} \approx T^{1.36} \text {. }
$$

The second observation is that data corresponding to similar conditions produce similarly shaped and closely grouped U-curves. The vocal and the letter data sets reported by Peterson and Peterson (1959), as well as the data set reported by Hellyer (1962), reflect similar conditions, in that the number of presentations variable $\left(X_{3}\right)$ in fact corresponds to time intervals filled with stimulus repetition at the rate of one per second. The computed phenomenological dimensions for these three data sets are $T^{1.55}, T^{1.36}$, and $T^{1.13}$, respectively. The silent data set reported by Peterson and Peterson produces a dramatically different curve-the leftmost one in Figure 3. The computed phenomenological dimension in this case is essentially different $\left(T^{4.80}\right)$. Moreover, the widely stretched U-curve does not really allow for specific determination of a dimension. It can be argued that, in this case, the number of presentations variable $\left(X_{3}\right)$ is of a different nature than in the other three cases, since it corresponds to time intervals filled with unspecified activities. (Recall that under the silent condition, the subjects were not given any specific instructions concerning this interval.)

Thus, although it seems evident that essentially different conditions result in essentially different values of phenomenological dimensions, can it be claimed that similar conditions result in similar values? Three arguments supporting the latter claim might be suggested.

Provided that the three phenomenological dimensions $T^{1.55}, T^{1.36}$, and $T^{1.13}$ (vocal, letter, and Hellyer's, 1962, data, respectively) correspond to similar psychological processes, one possible reason for the discrepancy can be sought in the inherently high variability in data of this sort caused by design and individual variability factors (Luce \& Krumhans1, 1988). Apparently, reasoning of this kind is applicable to these data sets, since they differ in both experimental design and subject pools.

A potential statistical reason for the discrepancy is the accuracy of estimation of the exponents in question, as calculated by the method of RDA. The relatively limited accuracy suggests an interpretation in terms of interval values, rather than single values, of the exponents in $T^{1.55}, T^{1.36}$, and $T^{1.13}$. As is shown in the bottom panel of Figure 3, all three curves overlap partially at about $C V / C V_{\min } \approx 1.08$. Thus, if the previously introduced ratio $\left(C V-C V_{\min }\right) / C V_{\min } \leq \delta^{*}$ is used as a criterion for determining interval values and an accuracy of about $\delta^{*}=10 \%$ is acceptable, which seems reasonable in the context of these data, the curves corresponding to the vocal, the letter, and Hellyer's (1962) data may be considered to reflect the same reality.

A final possibility for the discrepancy is the difference in the modality of stimulus presentation-visual (Hellyer, 1962) versus auditory (Peterson \& Peterson, 1959). Three psychophysical findings appear to be relevant to the effect of modality in the processing of time intervals. It has been shown that time intervals marked by auditory signals are judged to be longer than the same intervals marked by visual signals (Behar \& Bevan, 1961; Goldstone \& Goldfarb, 1963; Marks, 1978; Stevens \& Greenbaum, 1966). In addition, Goldstone and Goldfarb's study indicated that filled auditory intervals are judged to be longer than unfilled auditory intervals. Finally, Stevens and Greenbaum reported an increase in the exponent $n$ in $\Psi=C \Phi^{n}$ when filled auditory versus filled visual intervals are used. Thus, the exponents in the phenomenological dimensions corresponding to Peterson and Peterson's data ( $T^{1.55}$ and $\left.T^{1.36}\right)$ may differ from the exponent corresponding to Hellyer's data $\left(T^{1.13}\right)$, due to the effect of stimulus modality alone. Of course, it would be simplistic to directly implicate psychophysical findings (based on simple and constant stimuli) in a quantitative explanation of memory phenomena. Nevertheless, it appears to be reasonable to claim that if modality of presentation affects output in Peterson and Peterson's type of experiment, the effect has to be in the direction of increasing the value of the phenomenological dimension when auditory versus visual presentation is involved.

\section{GENERAL DISCUSSION}

The primary goal of this study was to show that DA can be used in analyzing psychological data in a similar way as in physics, provided that the dimensions of psychological variables are interpreted in the sense of RDA - a method that allows for extracting dimensions from data. A dimension obtained by this method, referred to as a phenomenological dimension, possesses properties that make it fundamentally different from a physical dimension. Therefore, before claiming the possibility for applications in psychophysics and, generally, in psychology, the first issue to be addressed is the legitimacy of both RDA per se and the dimensions obtained by this method. Provided that RDA is a consistent data analysis technique and the dimensions obtained by this method allow for meaningful interpretation, the second issue to be addressed is the prospect for use of the method in psychology.

\section{Legitimacy of RDA}

The RDA method raises three fundamental issues: (1) measuring quantities with unknown dimensions, (2) assigning dimensions to quantities, and (3) treating dimensional constants. The scope of this article, as well 
as the limited space, allows for only a brief introduction to these issues. Detailed consideration can be found in the following monographs: Bridgman (1922), Campbell (1920, 1921, 1928, 1952, 1957), Churchman and Ratoosh (1959), Ellis (1966), Krantz et al. (1971), Kyburg (1984), and McCrea and Rees (1983).

Measuring quantities with unknown dimensions. RDA deals with quantities with unclearly determined, or even unknown, dimensions. Can such quantities be measured? To our understanding, the answer to this question depends primarily on the definition of measurement used. Opinions in this matter are concentrated around two poles: the highly restrictive one commonly attributed to Campbell (1928), and the other, almost free of restrictions, attributed to Stevens (1959). A third alternative, more restrictive than Stevens' definition of measurement, but not as restrictive as Campbell's (1928), is provided by conjoint measurement theory (Krantz et al., 1971; Luce \& Krumhansl, 1988). According to Campbell (1928), the attribute being measured has to be additive in nature in order for the mapping onto a numerical scale to be recognized as measurement. As many measurement theorists have noted (cf. Krantz et al., 1971), such a requirement imposes severe restrictions on what can be considered as measurement. In fact, only a few well-known physical quantities, such as length and mass, meet this criterion. Thus, Campbell's (1928) definition of measurement does not leave much possibility, if any, for measuring something that is not clearly known in the sense of its physical properties, including dimensions. To the contrary, Stevens' (1959) definition of measurement, "assignment of numerals to objects or events according to rule-any rule ... provided a consistent rule is followed" (p. 19), allows for measuring virtually anything, provided a rule of mapping onto a numerical scale is established. A number of nonphysical disciplines, including psychology, have largely adopted the latter definition of measurement. The undiscriminating use of Stevens' definition of measurement in psychology was extensively criticized by Michell (1997) and Kline (1998). Both authors appealed for a return to a more restrictive definition of measurement (viz., scientific measurement). In essence, this approach assumes additivity, however, in a more abstract sense than the requirement for concatenation of physical attributes, as presumably was thought by Campbell (1928). In the case of psychological attributes, such additivity can be established (or refuted) by means of conjoint additive measurement (Krantz et al., 1971; Luce \& Krumhansl, 1988). There is no doubt that Stevens' widely adopted approach to measurement, by virtue of its definition, leaves the possibility for measuring attributes of an object or a phenomenon not clearly determined in terms of dimensions. It seems reasonable to assert that both scientific measurement, as defined by Michell, and conjoint additive measurement allow for measuring such attributes. In order to prove additivity, both approaches require less in-depth knowledge of the nature of the measured attribute than is required in the case of measurement in Campbell's (1928) sense. Neither these two approaches nor Campbell's (1928) approach explicitly requires knowledge of the dimension of an attribute in establishing (refuting) its measurability. Nevertheless, it is apparent that in the case of Campbell (1928), such knowledge is essential, whereas this is not so, or at least is less apparent, in the case of Michell and Krantz et al.

Assigning dimensions to quantities. As has already been noted, a physical dimension is viewed as a denotation (symbol or name) of a qualitatively distinguishable attribute of an object or a phenomenon and is defined prior to a particular measurement (Ellis, 1966; Krantz et al., 1971; Munson et al., 1990; Pankhurst, 1964). Can a phenomenological dimension, a hybrid (qualitativequantitative) entity defined after a particular measurement, be regarded as a dimension? A notion of dimension, referred to as a fractal dimension, which possesses properties similar to those of a phenomenological dimension, was introduced some 30 years ago in order to provide a consistent mathematical description of a class of geometrical objects called fractals (Mandelbrot, 1977, 1983). The most important achievement of Mandelbrot was showing that fractal dimensions provide the most adequate description of the geometrical properties of many complex objects and phenomena. A fractal dimension is a special case of a phenomenological dimension in which only the geometrical properties of an object or a phenomenon are considered (Marinov, 2001). It has been shown in geometry that fractal dimensions provide a meaningful description in cases in which Euclidean ones do not. It can be suggested by analogue that phenomenological dimensions may provide such a description in cases in which physical dimensions do not.

Treating dimensional constants. It is generally believed that dimensional constants are inherent to equations describing reality. This is because variables with different dimensions can be involved and, therefore, the only way to keep the equation dimensionally homogeneous is to add appropriate dimensional constants. If, for instance, $X_{1}$ and $X_{2}$ are two variables with different dimensions and it is found that $X_{1}$ is proportional to $X_{2}^{1.5}$, the corresponding equation will be written as $X_{1}=$ $C X_{2}^{1.5}$, where the constant $C$ with dimension $[C]=$ $\left[X_{1}\right] /\left[X_{2}\right]^{1.5}$ is introduced for the sole purpose of maintaining the dimensional homogeneity of the equation. RDA maintains the dimensional homogeneity of such equations by modifying dimensions in lieu of introducing dimensional constants. If the above relation between $X_{1}$ and $X_{2}$ is interpreted in the sense of RDA, no dimensional constant will be introduced, but the dimension of one of the variables-say, $X_{1}$ - will be modified from $\left[X_{1}\right]$ to $\left[X_{2}\right]^{1.5}$. It may be argued that, if all quantities are well known, such an interpretation makes little or no sense. In fact, even very familiar quantities, such as length, may exhibit properties that are more adequately interpreted in terms of RDA, rather than in terms of dimensional constants (Marinov, 2001). Moreover, Baren- 
blatt (1987) has shown that there are cases in which a dimensional constant cannot be introduced at all in order to have a dimensionally homogeneous equation. The only way to achieve this is to modify the dimension of the actual variable, as it occurs in both fractal geometry and RDA. Such an approach concurs with views that question the need of such constants (Bridgman, 1922), as well as their so-called "fundamental nature" (McCrea, 1983).

\section{Prospects for Use of RDA in Psychology}

The use of DA as a basis of RDA creates the impression that only ratio-scaled quantities are intended. In fact, RDA does not impose restrictions on problems involving any measurable quantities but may be used as a test for determining whether a quantity is ratio scaled.

The use of examples involving variables with physical dimensions may create the impression that RDA is applicable only if at least one of the variables relevant to the phenomenon being investigated has a physical dimension in the usual sense of this word (e.g., the dimension of time). In fact, RDA does not presume any requirements of the sort. By using appropriate statistical criteria, virtually any set of variables $X_{1}, X_{2}, \ldots X_{m}$ can be tested for the presence of a kind of relationship among these variables, which may be regarded as a dimension-like structure (DLS). DLS is thought of as a structure of relationships among any kind of quantities that is similar to those structures found among physical quantities when DA is applied. If such a structure is found, then, by analogue with physics, some of the variables may be regarded as variables with independent dimensions and others as variables with dependent dimensions. Furthermore, if variables of similar nature exhibit similar DLSs and a relatively stable pattern of dependent/independent dimensions when entering different functional relations, dimensions can be assigned to these variables, and such dimensions may be considered as intrinsic to the corresponding quantities. Such an approach may allow for developing a set of primary dimensions in the corresponding field, which will be completely independent of any physical dimensions and any physics in general. Such a set, however, will resemble the very essence of well-established physical notions and methods. In fact, De Jong (1967) in economics and Haynes (1982) in physical geography attempted to make use of DA by assigning dimensions to certain dimensionless variables (e.g., population size) and considering such dimensions as primary dimensions. The choice of such variables, however, was rather arbitrary and, therefore, not reflective of any real structure of relations among the quantities involved, except by coincidence.

The scheme of introducing primary dimensions is independent of both the kind of variables involved and the specific field the variables belong to. Therefore, such a scheme is applicable in all three cases: (1) a set of nonphysical variables only, (2) a set consisting of both nonphysical and physical variables, and (3) a set of physical variables only. The three cases are exemplified by (1) psychomet- rics, (2) psychophysics, and (3) physics involving fractal shapes, respectively.

The importance of developing the notion of primary (basic) dimensions in psychology has been emphasized by Ward (1992). To our knowledge, the developing of such a notion is viewed largely as a problem of establishing a link of the sort found in physics between the psychological and the physical realms. For instance, in the context of measuring psychological magnitudes, Luce and Krumhansl (1988) note the following: "For the measurement view to prevail . . . it appears necessary for some structure of interconnected scales to arise, much as exist in physics. This would mean a complex pattern of reductions of some psychological scales to others, with a resulting simple pattern of units, and quite possibly some simple connection with the scales of physics" (pp. 4-5). Ward suggests that the psychological space, as defined by Shepard (1987), can be used as a linkage between the psychological and the physical, since the organization of the objects within the space (represented by distances) is considered to be of a psychological nature, whereas the dimensions of such a space are considered to be physical. A major obstacle in this matter, however, appears to be the fact that the psychological is not defined in terms of any dimensions that appear in the physical (Van Brakel, 1993, cited in Marks \& Algom, 1998, p. 134).

Within the framework of RDA, the problem of linking the psychological and the physical in a psychophysical context is naturally resolved by assigning phenomenological dimensions to psychological variables and then treating them on a par with the genuine physical variables. In the context of psychology, in cases in which no physical variables are involved, the question of linking with the physical may not be raised at all, in the sense of an inevitable intermediate step in introducing psychological dimensions. Such dimensions can be assigned to the psychological variables by the means of RDA, provided DLSs are found. This approach not only resembles well-established physical notions and methods, as was noted above, but also allows for the use of the convenient language of physics until an appropriate language is developed.

\section{Concluding Remarks}

The complexity of psychological phenomena appears to be the major cause of the lack of irrefutable quantitative models describing such phenomena in terms of simple functions of a few parameters. Even in psychophysics, such models are subject to criticism (Baird, 1997; Luce \& Krumhansl, 1988; Marks, 1974; Marks \& Algom, 1998; West, Ward, \& Khosla, 2000). Stevens' law, $\Psi=C \Phi^{n}$, is criticized because of deviations from a power function, as well as high variability in the exponent $n$ due to factors not related to variations in the stimulus $\Phi$. Anderson $(1975,1992)$ considered this law to be a conceptual misdirection because of both the use of a simple function across all sensory modalities and the in- 
capability of handling such aspects of sensation as context and hedonics. Michell (1997) has questioned even the very foundation of quantitative psychology-the quantitative nature of psychological entities, including sensations.

RDA faces the usual risk of being perceived as just another attempt to reduce the whole diversity of psychological phenomena to a colorless quantitative structure. What, in fact, makes this approach different from many others proposed in quantitative psychology is presumably the deeper level of consideration - the level of dimensions of quantities. Bridgman (1922, p. 52) regards DA as "an analysis of an analysis." Such an analysis deals with most fundamental concepts and imposes restrictions affecting upper levels, including those of admissible functional relations among variables. If this is the case with RDA, the method might be capable of capturing the essence of the quantitative aspects of a psychological phenomenon, if such aspects are present at all. On the other hand, RDA might serve as a test for the very existence of such aspects, since it resembles a well-established and indisputable quantitative method in physics.

\section{REFERENCES}

ANDERSON, N. H. (1975). On the role of context effects in psychophysical judgment. Psychological Review, 82, 462-482.

ANDERSON, N. H. (1992). Integration psychophysics and cognition. In D. Algom (Ed.), Psychophysical approaches to cognition (pp. 13-113). Amsterdam: North-Holland.

BAIRD, J. C. (1997). Sensation and judgment: Complementary theory of psychophysics. Mahwah, NJ: Erlbaum.

BARE NBlatt, G. I. (1987). Dimensional analysis. New York: Gordon \& Breach.

BEHAR, I., \& BEVAN, W. (1961). The perceived duration of auditory and visual intervals: Cross modal comparison and interaction. American Journal of Psychology, 74, 17-26.

Bridgman, P. (1922). Dimensional analysis. New Haven, CT: Yale University Press.

BUCKINGHAM, E. (1914). On physically similar systems: Illustrations of the use of dimensional equations. Physical Review, 4, 345-376.

Campbell, N. (1920). Physics: The elements. Cambridge: Cambridge University Press.

CAMPBELL, N. (1921). What is science? London: Methuen.

CAMPBELL, N. (1928). An account of the principles of measurement and calculation. London: Longmans, Green \& Co.

CAMPBell, N. (1952). What is science? New York: Dover. (Original work published 1921)

CAMPBELl, N. (1957). Foundations of science: The philosophy of theory and experiment. New York: Dover. (Original work published as Physics: The elements, 1920)

Churchman, C. W., \& Ratoosh, P. (Eds.) (1959). Measurement: Definitions and theories. New York: Wiley.

Collins, G. S. (1984). Renormalization. Cambridge: Cambridge University Press.

Creswick, R. J., FARACH, H. A., \& PoOle, C. P., JR. (1992). Introduction to renormalization group methods in physics. New York: Wiley.

DANIEL, C., \& WoOD, F. S. (1971). Fitting equations to data: Computer analysis of multifactor data for scientists and engineers. New York: Wiley.

DE Jong, F. J. (1967). Dimensional analysis for economists. Amsterdam: North-Holland.

Ellis, B. (1966). Basic concepts of measurement. Cambridge: Cambridge University Press.

FECHNER, G. T. (1966). Elements of psychophysics (Vol. 1; H. I. Adler,
Trans.). New York: Holt, Rinehart \& Winston. (Original work published 1860)

Goldstein, E. B. (1989). Sensation and perception. Belmont, CA: Wadsworth.

Goldstone, S., \& GoldFARB, J. (1963). Judgment of filled and unfilled durations: Intersensory effects. Perceptual \& Motor Skills, 17, 763-774.

HARNETT, D. L., \& MURPHY, J. L. (1993). Statistical analysis for business and economics. Don Mills, ON: Addison-Wesley.

HAYNES, R. M. (1982). An introduction to dimensional analysis for geographers. Norwich: Geo Abstracts.

HeLLYER, S. (1962). Frequency of stimulus presentation and short-term decrement in recall. Journal of Experimental Psychology, 64, 650.

KLINE, P. (1998). The new psychometrics: Science, psychology, and measurement. London: Routledge.

Krantz, D. H., Luce, R. D., Suppes, P., \& TVersky, A. (1971). Foundations of measurement: Vol. I. Additive and polynomial representations. New York: Academic Press.

Kyburg, H. E. (1984). Theory and measurement. Cambridge: Cambridge University Press.

LAMING, D. (1992). Analysis of short-term retention. Journal of Experimental Psychology: Learning, Memory, \& Cognition, 18, 1342-1365.

LAMING, D. (1997). The measurement of sensation. Oxford: Oxford University Press.

LuCE, R. D. (1959). On the possible psychophysical laws. Psychological Review, 66, 81-95.

LUCE, R. D. (1964). A generalization of a theorem of dimensional analysis. Journal of Mathematical Psychology, 1, 278-284.

LUCE, R. D. (1971). Similar systems and dimensionally invariant laws. Philosophy of Science, 38, 157-169.

LUCE, R. D. (1978). Dimensionally invariant numerical laws correspond to meaningful qualitative relations. Philosophy of Science, 45, 1-16.

LUCE, R. D. (1990). 'On the possible psychophysical laws' revised: Remarks on cross-modal matching. Psychological Review, 97, 66-77.

LucE, R. D., \& KRumhansL, C. L. (1988). Measurement, scaling, and psychophysics. In R. C. Atkinson et al. (Eds.), Stevens' handbook of experimental psychology (Vol. 1, pp. 3-74). New York: Wiley.

MANDElbRot, B. (1977). Fractals: Form, chance and dimension. San Francisco: Freeman.

MANDELBROT,B. (1983). The fractal geometry of nature. San Francisco: Freeman.

MARINOV, S. A. (1994). On a reversed process of dimensional analysis: Theory. In L. M. Ward (Ed.), Proceedings of the 10th Annual Meeting of the International Society for Psychophysics (pp. 131-136). Vancouver, BC: International Society for Psychophysics.

MARINOv, S. A. (1999). Dimensions as quantities. In B. Kroplin, S. Rudolph, \& S. Bruckner (Eds.), Proceedings of the 2nd International Workshop on Similarity Methods (pp. 111-127). Stuttgart: Stuttgart University Press.

MARINOV, S. A. (2001). Fractal dimension as a special case of phenomenological dimension. In B. Kroplin, S. Rudolph, \& S. Bruckner (Eds.), Proceedings of the 4th International Workshop on Similarity Methods (pp. 117-127). Stuttgart: Stuttgart University Press.

MARINOV, S. A., \& PANOv, A. (2000). Reversed dimensional analysis: An alternative way of estimating constants in psychophysics. In C. Bonnet (Ed.), Proceedings of the 16th Annual Meeting of the International Society for Psychophysics (pp. 257-262). Strasbourg, France: International Society for Psychophysics.

MARKs, L. E. (1974). Sensory processes: The new psychophysics. New York: Academic Press.

MARKS, L. E. (1978). The unity of the senses: Interrelations among the modalities. New York: Academic Press.

MARKs, L. E. (1992). 'What thin partitions sense from thought divide': Toward a new cognitive psychophysics. In D. Algom (Ed.), Psychophysical approaches to cognition (pp. 115-186). Amsterdam: NorthHolland.

Marks, L. E., \& Algom, D. (1998). Psychophysical scaling. In M. H. Birnbaum (Ed.), Measurement, judgment, and decision making (pp. 81-178). San Diego: Academic Press.

MCCREA, W. H. (1983). Introductory remarks. In W. H. McCrea \& M. J. 
Rees (Eds.), The constants of physics. Philosophical Transactions of the Royal Society of London: Series A, 310, 211-213.

MCCREA, W. H., \& REES, M. J. (EDS.) (1983). The constants of physics [Special issue]. Philosophical Transactions of the Royal Society of London: Series A, 310 (1512), 209-363.

MiCHELL, J. (1997). Quantitative science and the definition of measurement in psychology. British Journal of Psychology, 88, 355-383.

Munson, B. R., Young, D. F., \& OKIISHI, T. H. (1990). Fundamentals offluid mechanics. New York: Wiley.

PANKHURST, R. C. (1964). Dimensional analysis and scale factors. New York: Reinhold.

Peterson, L. R., \& Peterson, M. J. (1959). Short-term memory retention of individual verbal items. Journal of Experimental Psychology, 58, 193-198.

SEDOv, L. I. (1981). Metody podobiya i razmernosti v mekhanike. [Similarity and dimensional methods in mechanics]. Moscow: Nauka. [In Russian]
SHE PARD, R. N. (1987). Toward a universal law of generalization for psychological science. Science, 237, 1317-1323.

STEVENS, S. S. (1959). Measurement, psychophysics, and utility. In C. W. Churchman \& P. Ratoosh (Eds.), Measurement: Definitions and theories (pp. 18-63). New York: Wiley.

Stevens, S. S. (1975). Psychophysics: Introduction to its perceptual, neural and social properties. New York: Wiley.

Stevens, S. S., \& Greenbaum, H. B. (1966). Regression effect in psychophysical judgment. Perception \& Psychophysics, 1, 439-446.

VAN BRAKEL, J. (1993). The analysis of sensations as the foundation of all sciences. Behavioral \& Brain Sciences, 16, 163-164.

WARD, L. M. (1992). Mind in psychophysics. In D. Algom (Ed.), Psychophysical approaches to cognition (pp. 187-249). Amsterdam: North-Holland.

West, R. L., WARD, L. M., \& Khosla, R. (2000) Constrained scaling: The effect of learned psychophysical scales on idiosyncratic response bias. Perception \& Psychophysics, 62, 137-151.

\section{APPENDIX}

This appendix is a short introduction to the method of RDA. Two illustrative examples of dealing with twovariable and three-variable problems with one of the variables having an unknown dimension will be used. Besides having simplicity, these two special cases will be considered since they formally correspond to Stevens' psychophysical law and Hellyer's (1962) and Peterson and Petersons's (1959) data, respectively, which have been analyzed in the main text. The general case of $m$-variable problems is similar in essence to the three-variableproblem, except for the use of much heavier mathematical expressions (Marinov, 1999). The more important of them will be quoted at the end of this Appendix.

\section{Case of Two Variables}

Consider a study of a phenomenon that is described by two variables only. Let no reasons be found to introduce any dimensional constants. In such a case, the functional relation between these two variables - say, $X_{1}$ and $X_{2}$ - can be written as

$$
f\left(X_{1}, X_{2}\right)=0
$$

or

$$
X_{1}=f\left(X_{2}\right)
$$

where $f$ stands for any functional relation.

Within the framework of DA (cf. Barenblatt, 1987), such a relation is physically possible if and only if the dimension of one of the variables depends on the dimension of the other variable. In such a case, according to the $\Pi$-theorem of DA, the relation between these two dimensional variables $(m=2)$ can be reduced to only one dimensionless variable $(m-k=2-1=1)$, as follows:

$$
\Pi_{1}=C,
$$

where $C$ is a dimensionless constant, and $\Pi_{1}$ is a dimensionless product of powers of the two variables:

$$
\Pi_{1}=X_{1}^{p_{1}} X_{2}^{p_{2}} .
$$

The dimensionless variable $\Pi_{1}$ in Equation A4 can be redefined (e.g., by raising both sides to the power of $1 / p_{1}$ ) so that only one exponent appears on the right side:

$$
\Pi_{1}=X_{1} X_{2}^{p} \text {. }
$$

As far as $\Pi_{1}$ is dimensionless, the relation between the dimensions of the two dimensional variables $X_{1}$ and $X_{2}$ is given by the expression

$$
\left[X_{1}\right]=\left[X_{2}\right]^{-p} .
$$

When both variables have known dimensions, the task of determining the exponent $p$ in Equation A5 is elementary.

For instance, if the first variable is the area of a circle, and if the second variable is the radius of the circle, the dimensions of the variables are $\left[X_{1}\right]=L^{2}$ and $\left[X_{2}\right]=L$, where $L$ stands for the dimension of length. In 
such a case, it is obvious by inspection that $p=-2$ in order for the dimension of $X_{2}$ to cancel the dimension of $X_{1}$ in Equation A5: $\left[X_{1} X_{2}^{p}\right]=L^{2}(L)^{-2}=L^{2} / \mathrm{L}^{2}=1$, where 1 denotes the dimension of a dimensionless quantity. Needless to say, such reasoning, based on DA, results in the formula $X_{1}=C X_{2}^{2}$, or in more familiar denotations, $A=\pi r^{2}$, which is the well-known expression for the area of a circle. The numerical value of the dimensionless constant $\pi$ cannot be found by means of DA alone.

A more complex problem arises when one of the variables in Equation A5 has an unknown dimension. As was previously discussed, not only is it possible that a variable with unknown dimension can be measured, but this is, in fact, the common case in psychology and especially in psychophysics, where such variables are put together in a functional relation along with variables with clearly defined physical dimensions.

If one of the variables in Equation A5 has unknown dimension, the exponent $p$ cannot be found as it can be in the case in which both variables have known dimensions. Moreover, $p$ cannot be found at all unless measurement data with respect to both variables are available. If, however, such data are available, the expression in Equation A5 can be rewritten in the form

$$
X_{1}=C X_{2}^{-p},
$$

and the problem of determining $p$ can be reduced to a linear regression problem:

$$
\ln \left(X_{1}\right)=\ln C+(-p) \ln \left(X_{2}\right) .
$$

If, for instance, the dimension of the first variable, $X_{1}$, is considered unknown, it can be determined, literally calculated, by substituting the numerical value of $p$ in Equation A6.

The dimension of $X_{1}$, as defined above, differs essentially from a physical dimension in at least two aspects: (1) Its exponent is a quantity that need not be an integer, and (2) it is unknown prior to measurement. Such a dimension, referred to as the phenomenological dimension (Marinov, 1999), is similar in nature to a fractal dimension - a fundamental concept in fractal geometry (Mandelbrot, 1983). Unlike a fractal dimension, a phenomenological dimension is not limited to the description of only the geometrical properties of the object or phenomenon being investigated.

As has been shown above, calculating phenomenological dimensions by means of RDA involves regression analysis, which is statistical in nature. Therefore, in the first place, statistical variations will be inherent to the method of RDA. In the second place, various statistical characteristics can be used in estimating the exponent $p$ as a statistical entity. For instance, if the coefficient of variation for regression residuals, $C V$ (cf. Harnett $\&$ Murphy, 1993), is used as a goodness-of-fit criterion with respect to Equation A8, a condition of the kind $C V \leq \delta$, where $\delta$ is a small number chosen a priori, can be imposed in order to accept (or refute) $p$ in a statistical sense. Obviously, the larger the value of $C V$, the more significant the departure from a linear function in the case of Equation A8 or from a power function in the case of Equation A7. Apart from statistical variations, within the framework of DA (respectively, RDA) such large deviations can be considered as a sign of improper initial choice of the kind and number of dimensional variables/constants relevant to the object or phenomenon being investigated.

Table A1

Four Possible Kinds of Combinations of Dimensions That Preserve the Dimensional Homogeneity of Functional Relation (A9)

\begin{tabular}{cccccc}
\hline Case & $x_{1}=\left[X_{1}\right]$ & $x_{2}=\left[X_{2}\right]$ & $x_{3}=\left[X_{3}\right]$ & $N_{k}$ & $N_{\Pi}$ \\
\hline 1 & 1 & 1 & 1 & 0 & 3 \\
2 & 1 & $x_{2}$ & $x_{3}=\varphi\left(x_{2}\right)$ & 1 & 2 \\
3 & $x_{1}=\varphi\left(x_{2}\right)$ & $x_{2}$ & $x_{3}=\varphi\left(x_{2}\right)$ & 1 & 2 \\
4 & $x_{1}=\varphi\left(x_{2}, x_{3}\right)$ & $x_{2}$ & $x_{3}$ & 2 & 1 \\
\hline
\end{tabular}

Note-Small $x$ s stand for the dimensions of the corresponding variables denoted by capital $X \mathrm{~s}$. The Greek letter $\varphi$ symbolizes that the corresponding dimension depends on (is a function of) the dimension(s) in parentheses. No specific functional relation is meant. $N_{k}$ stands for the number of variables with independent dimensions, whereas $N_{\Pi}$ stands for the number of dimensionless $\Pi$-terms. *The dimension of a dimensionless quantity is not considered as an independent dimension, since it can always be expressed as a ratio of any independent dimension to itself (Barenblatt, 1987). 


\section{APPENDIX (Continued)}

\section{Case of Three Variables}

Consider a study of a phenomenon that is described by three variables only. Let no reasons be found to introduce any dimensional constants. In such a case, the functional relationship between these variables-say, $X_{1}, X_{2}$, and $X_{3}$ - can be written as

$$
f\left(X_{1}, X_{2}, X_{3}\right)=0 \text {. }
$$

Within the framework of DA, such a relation is physically possible if and only if one of the four kinds of combinations of dimensions shown in Table A1 is present. Obviously, rearranging (renumbering) the variables within any of these four kinds of combinations will produce other admissible combinations belonging to the same kind.

Consider, for instance, Case 2 in Table A1. According to the П-theorem of DA, the relation between these three dimensional variables can be reduced to a relation between two dimensionless variables ( $\Pi$-terms) as follows:

$$
\Pi_{1}=F\left(\Pi_{2}\right),
$$

where the Пs are dimensionless products of powers of the three variables:

and

$$
\Pi_{1}=X_{1}^{p_{11}} X_{2}^{p_{12}} X_{3}^{p_{13}}
$$

$$
\Pi_{2}=X_{1}^{p_{21}} X_{2}^{p_{22}} X_{3}^{p_{23}} \text {. }
$$

There exists a general method for determining the exponents in the expressions for the $\Pi$-terms, referred to as the method of recurrent variables (see, e.g., Munson et al., 1990). In the case of Equation A11, however, the exponents can be found simply by inspection. Taking into account the dimensions of the variables shown in Table A1, the following relations hold:

$$
\begin{aligned}
& \Pi_{1}=X_{1}^{1} X_{2}^{0} X_{3}^{0}, \\
& \Pi_{2}=X_{1}^{0} X_{2}^{p_{22}} X_{3}^{p_{23}}
\end{aligned}
$$

or

$$
\begin{aligned}
& \Pi_{1}=X_{1}, \\
& \Pi_{2}=X_{2}^{p_{22}} X_{3}^{p_{23}} .
\end{aligned}
$$

As in the case of two variables, $\Pi_{2}$ can be redefined (e.g., by raising both sides to the power of $1 / p_{23}$ ), so that only one exponent appears on the right side:

$$
\begin{aligned}
& \Pi_{1}=X_{1}, \\
& \Pi_{2}=X_{2}^{p} X_{3},
\end{aligned}
$$

and then Equation A10 can be rewritten in the form

$$
X_{1}=F\left(X_{2}^{p} X_{3}\right),
$$

where the relation between the dimensions of the two variables $X_{2}$ and $X_{3}$ is given by the expression

$$
\left[X_{3}\right]=\left[X_{2}\right]^{-p} \text {. }
$$

Similar to the case of two variables, if one of the variables in Equation A9 has an unknown dimension, the exponent $p$ cannot be found at all, unless measurement data for all three variables are available. Unlike in the case of two variables ( see Equation A8), even when such data are available, the problem of determining $p$ cannot be reduced to a linear regression problem, since the explicit form of Equation A14 is unknown. It can be found, however, via multiple nonlinear regression analysis, where the set of regression parameters will also include the unknown exponent $p$. For instance, in order to find an approximation to Equation A14 according to the method of least squares (cf. Harnett \& Murphy, 1993), the following sum has to be minimized:

$$
\sum\left[X_{1}-F_{0}\left(X_{2}^{p} X_{3}\right)\right]^{2}
$$

where $F_{0}$ is a regression function selected a priori. 


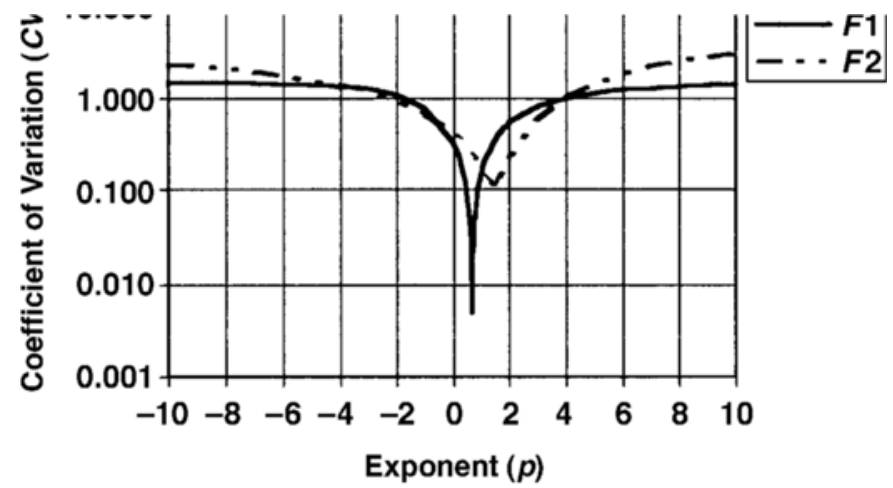

Figure A1. The coefficient of variation for regression residuals $(\mathrm{CV})$ as a function of the exponent $p$ within the interval $-10 \leq p \leq+10$ for two regression functions, $F 1$ and $F 2$. Model data.

Apparently, the sum in Equation A16 is a function of both the regression parameters per se and the unknown exponent $p$. If this function is denoted by $\Omega$, both the regression parameters and the exponent $p$ can be found as a solution to the following system of equations:

$$
\begin{aligned}
& \frac{\partial}{\partial C_{i}} \Omega\left(C_{1} \ldots C_{r}, p\right)=0, \quad(i=1 \ldots r) \\
& \frac{\partial}{\partial p} \Omega\left(C_{1} \ldots C_{r}, p\right)=0
\end{aligned}
$$

where $C_{1} \ldots C_{r}$ are regression parameters and $r$ is the number of such parameters, which depends on the particular form of the selected regression function $F_{0}$.

In turn, substituting $C_{1} \ldots C_{r}$ and $p$ in Equation $\mathrm{A} 14$ allows for calculating various statistical characteristics and numerically estimating the goodness of fit by the selected regression function $F_{0}$. Following this procedure, a variety of regression functions - say, $F_{0}^{(1)}, F_{0}^{(2)}, \ldots F_{0}^{(q)}$ - can be tested, and the one that provides the best fit can be chosen. The numerical value of $p$ that corresponds to this function can then be substituted into Equation A15 in order to find the dimension of variable $X_{3}$.

Note that this procedure of choosing the best-fitting function is not much different from the one used in regression analysis, with the exception that the complete set of regression parameters also includes the unknown exponent $p$. In practice, another difference between the procedure described in this article and the one commonly used in regression analysis is that, in the former case, the choice of an appropriate regression function cannot be facilitated by visual inspection of plotted data, since different values of $p$ may produce essentially different patterns of data plotted in the same coordinates $\Pi_{1}=F\left(\Pi_{2}\right)$.

Since Equation A17 is essentially nonlinear with respect to $p$, the system of equations may have more than one solution or may not have a solution at all. Generally, even a few discrete values of $p$, solutions to Equation A17, will complicate the choice of the value to be used in assigning a phenomenological dimension to the variable $X_{3}$ in Equation A15. Under these circumstances, it appears useful to look not only for a single value of the exponent $p$ by solving Equation A17 but also for an interval of values within some margins-say, $p_{a} \leq p \leq p_{b}$. Accordingly, if each value of $p$ that belongs to the interval $\left(p_{a}, p_{b}\right)$ is substituted into Equation A17 and the regression parameters $C_{1} \ldots C_{r}$ for a given regression function $F_{0}$ are found, the goodness of fit for this particular function can be estimated across the entire interval $\left(p_{a}, p_{b}\right)$. If, for instance, the coefficient of variation for regression residuals $(C V)$ is used as a goodness-of-fit criterion, a complete picture of the function $C V=C V(p)$ within the interval $\left(p_{a}, p_{b}\right)$ can be found. Such a picture will show all local minima, if any, including the absolute minimum for this interval. If such an absolute minimum exists and does not coincide with one of the margins of the interval $\left(p_{a}, p_{b}\right)$, the value of $p=p_{\min }$ that corresponds to $C V_{\min }=C V\left(p_{\min }\right)$ will be used in assigning a phenomenological dimension to the variable $X_{3}$ in Equation A15.

Figure $\mathrm{A} 1$ illustrates the above reasoning in the case of two regression functions denoted by $F 1$ and $F 2$. In both cases, $C V=C V(p)$ has a single minimum within the interval $-10 \leq p \leq+10$. However, $C V_{\min }^{(F 1)}<C V_{\min }^{(F 2)}$, 


\section{APPENDIX (Continued)}

or in other words, $F 1$ provides a better fit than does $F 2$. Therefore, the value of $p$ that corresponds to $C V_{\mathrm{min}}^{(F 1)}($ in Figure $\mathrm{A} 1, p_{\min }^{(F 1)} \approx 0.67$ and $\left.C V_{\min }^{(F 1)} \approx 0.0049\right)$ will be used in assigning a phenomenological dimension to the variable $X_{3}$ in an expression of the kind in Equation A15.

As was emphasized above, statistical variations will be inherent to the method of RDA, for it involves regression analysis. In the case of three variables, statistical criteria, such as $C V \leq \delta$, can be used not only in making a decision as to whether $p$ is acceptable in a statistical sense, but also, as was just illustrated, in choosing the regression function that provides the best fit among all the functions considered.

\section{General Case}

The procedure of obtaining (calculating) phenomenological dimensions in the case of $m$-variable problems is similar in its essence to the three-variableproblem. To avoid details, if one of the variables-say, $X_{l}$, in $f\left(X_{1}\right.$, $\left.X_{2}, \ldots X_{m}\right)=0$ has an unknown dimension, the dimension of this variable is given by the expression

$$
\left[X_{l}\right]=\left[X_{1}\right]^{-p_{l-k, 1}}\left[X_{2}\right]^{-p_{l-k, 2}} \ldots\left[X_{k}\right]^{-p_{l-k, k}},
$$

where $k$ is the number of variables with independent dimensions and the exponents $p_{l-k, 1} \ldots p_{l-k, k}$ are found as solutions to the system

$$
\begin{array}{ll}
\frac{\partial}{\partial C_{i}} \Omega\left(C_{1} \ldots C_{r}, p_{l-k, 1} \ldots p_{l-k, k}\right)=0, & (i=1 \ldots r) \\
\frac{\partial}{\partial p_{l-k, j}} \Omega\left(C_{1} \ldots C_{r}, p_{l-k, 1} \ldots p_{l-k, k}\right)=0, & (j=1 \ldots k),
\end{array}
$$

where $\Omega=\Sigma\left[\left(\Pi_{1}-F_{0}\left(\Pi_{2}, \Pi_{3}, \ldots \Pi_{l-k} \ldots \Pi_{n-k}\right)\right]^{2}, F_{0}\right.$ is a selected regression function, $C_{1} \ldots C_{r}$ are regression parameters, $r$ is the number of such parameters, and all $\Pi$-terms are dimensionless products of powers of the dimensional variables

$$
\Pi_{i}=X_{1}^{p_{i, 1}} \quad X_{2}^{p_{i, 2}} \ldots X_{k}^{p_{i, k}} X_{k+i}, \quad i=1 \ldots k .
$$

It is assumed that the variables are rearranged (renumbered) in such a way that the first $k$ variables are those with independent dimensions.

Readers interested in a more detailed discussion of the $m$-variable problem may consult Marinov (1999).

(Manuscript received August 25, 2000;

revision accepted for publication May 7, 2003.) 